\title{
Influence of the fermionic exchange symmetry beyond Pauli's exclusion principle
}

\author{
Felix Tennie, ${ }^{1}$ Vlatko Vedral, ${ }^{1,2}$ and Christian Schilling ${ }^{1, *}$ \\ ${ }^{1}$ Clarendon Laboratory, University of Oxford, Parks Road, Oxford OX1 3PU, United Kingdom \\ ${ }^{2}$ Centre for Quantum Technologies, National University of Singapore, 3 Science Drive 2, Singapore 117543 \\ (Received 2 September 2015; revised manuscript received 30 December 2015; published 24 February 2017)
}

\begin{abstract}
Pauli's exclusion principle has a strong impact on the properties of most fermionic quantum systems. Remarkably, the fermionic exchange symmetry implies further constraints on the one-particle picture. By exploiting those generalized Pauli constraints, we derive a measure which quantifies the influence of the exchange symmetry beyond Pauli's exclusion principle. It is based on a geometric hierarchy induced by the exclusion principle constraints. We provide a proof of principle by applying our measure to a simple model. In that way, we conclusively confirm the physical relevance of the generalized Pauli constraints and show that the fermionic exchange symmetry can have an influence on the one-particle picture beyond Pauli's exclusion principle. Our findings provide a perspective on fermionic multipartite correlation since our measure allows one to distinguish between static and dynamic correlations.
\end{abstract}

DOI: 10.1103/PhysRevA.95.022336

\section{INTRODUCTION}

The properties and the behavior of most fermionic quantum systems strongly rely on Pauli's famous exclusion principle [1]. This principle defines a constraint on the one-particle picture: For any $N$-fermion state $\left|\Psi_{N}\right\rangle$, the occupancies of one-particle states $|\varphi\rangle$ are restricted, $0 \leqslant\left\langle\Psi_{N}\left|\hat{n}_{\varphi}\right| \Psi_{N}\right\rangle \leqslant 1$. Indeed, this constrains the one-particle reduced density matrix $\rho_{1} \equiv N \operatorname{tr}_{N-1}\left[\left|\Psi_{N}\right\rangle\left\langle\Psi_{N}\right|\right]$, obtained by tracing out the $N-1$ fermion, according to

$$
\mathbb{0} \leqslant \rho_{1} \leqslant \mathbb{1} \text {. }
$$

Equivalent to this matrix relation, the natural occupation numbers $\lambda_{i}$, i.e., the eigenvalues of the one-particle reduced density matrix, are restricted,

$$
0 \leqslant \lambda_{i} \leqslant 1 \text {. }
$$

These Pauli constraints play an important role for various physical phenomena, with remarkable consequences for both the micro- and the macroworld. On a microscopic scale, they are the basis of the "Aufbau principle" for atoms and nuclei. For macroscopic systems, the Pauli exclusion principle is responsible for the stability of matter [2,3]. In particular, for an ideal gas of fermions, it implies an effective pressure, i.e., the Fermi degeneracy pressure, explaining on a rudimentary level the stability of neutron stars. Further emerging phenomena are the Fermi hole and the Pauli spin blockade. They originate from the fact that electrons with parallel spins are forbidden to sit at the same position.

This universal relevance of Pauli's exclusion principle is obvious for weakly correlated systems: all Pauli constraints are (approximately) saturated, i.e., one observes for each occupation number either $\lambda_{i} \approx 1$ or $\lambda_{i} \approx 0$. Such (approximate) pinning by all Pauli constraints is the typical behavior within the Landau-Fermi theory. Even for strongly correlated systems, one observes this quasipinning by Pauli constraints since at least the largest occupation numbers are very close to one and the smallest ones are very close to zero.

\footnotetext{
${ }^{*}$ christian.schilling@ @ physics.ox.ac.uk
}

Despite the success on its own, the Pauli constraints in the one-particle picture are de facto a consequence of the antisymmetry of the $N$-fermion wave function [4,5]. This exchange symmetry is a much stronger requirement than Pauli's exclusion principle concerning the one-particle picture only. It has been crucial, e.g., for the heuristic approach to the fractional quantum Hall effect. Using a Jastrow-type wave function involving a product of antisymmetric two-particle functions $\left(z_{i}-z_{j}\right)^{p}$ with $p$ an odd, positive integer, Laughlin succeeded to explain a huge class of plateaus with fractional Hall conductivity [6].

Since the exchange symmetry concerns the $N$-particle picture, an important question arises: Does the fermionic exchange symmetry have an influence on one-particle properties beyond the Pauli exclusion principle? Until a few years ago, it has even been unclear how to address this question in a meaningful way for concrete systems. This has changed thanks to a recent mathematical breakthrough. Motivated by results for a very few special cases [7], it was shown that the fermionic exchange symmetry in general implies further restrictions on the one-particle picture [8-10], completing Pauli's incomplete exclusion principle. Those generalized Pauli constraints will serve us to eventually address in the present work the longstanding question above. The corresponding findings exploiting an elegant quantum information theoretical language will provide a perspective on fermionic multipartite correlation since they allow the distinction between static and dynamic correlation.

\section{GENERALIZED PAULI CONSTRAINTS}

The so-called generalized Pauli constraints take the form of linear inequalities,

$$
D_{j}(\vec{\lambda}) \equiv \kappa_{j}^{(0)}+\sum_{i=1}^{d} \kappa_{j}^{(i)} \lambda_{i} \geqslant 0,
$$

for the natural occupation numbers $\lambda_{i}$. These are the (decreasingly ordered) eigenvalues of the one-particle reduced density matrix corresponding to the $N$-fermion state $\left|\Psi_{N}\right\rangle$. Here, $\kappa_{j}^{(i)} \in \mathbb{Z}, j=1,2, \ldots, v^{(N, d)}<\infty$, and $d$ is the dimension 


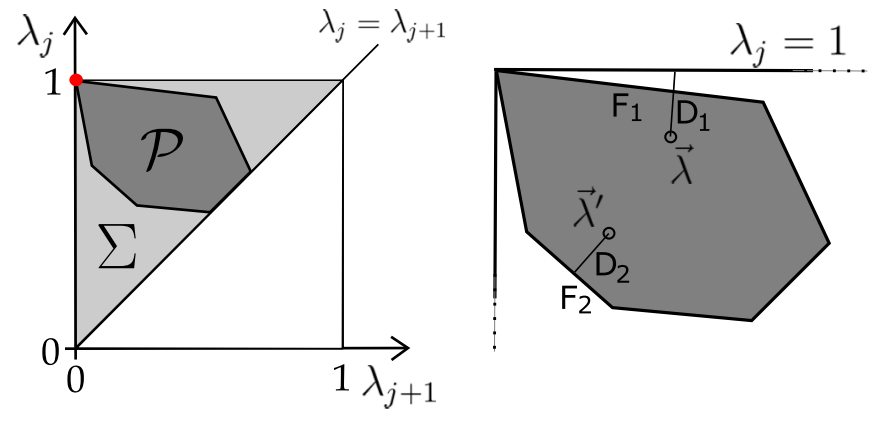

FIG. 1. Left: Schematic illustration of the polytope $\mathcal{P}$, which is a proper subset of the light-gray "Pauli simplex" $\Sigma$. The HartreeFock point is shown as a red dot. Right: For some generalized Pauli constraints ( $D_{1}$ but not $D_{2}$ ), a small distance of $\vec{\lambda}$ to the boundary of the surrounding Pauli simplex enforces a small distance $D_{j}(\vec{\lambda})$ of $\vec{\lambda}$ to the polytope facet $F_{j}$.

of the underlying one-particle Hilbert space. Geometrically, for each fixed pair $(N, d)$, the family of generalized Pauli constraints, together with the normalization and the ordering constraints $\lambda_{1} \geqslant \cdots \geqslant \lambda_{d} \geqslant 0$, defines a $(d-1)$-dimensional polytope $\mathcal{P}^{(N, d)}$ of allowed vectors $\vec{\lambda} \equiv\left(\lambda_{i}\right)_{i=1}^{d}$.

Each of the $D_{j}(\vec{\lambda})$ determines a $(d-2)$-dimensional facet $\mathcal{F}_{j}^{(N, d)}$ of $\mathcal{P}^{(N, d)}$,

$$
\mathcal{F}_{j}^{(N, d)} \equiv\left\{\vec{\lambda} \in \mathcal{P}^{(N, d)} \mid D_{j}(\vec{\lambda})=0\right\} .
$$

The remaining facets of $\mathcal{P}^{(N, d)}$ are defined by the saturation of the "ordering constraints," i.e., by $\lambda_{i}=\lambda_{i+1}$ for $i=1, \ldots$, $d-1$.

To compare the generalized Pauli constraints with the Pauli constraints, notice that the Pauli constraints (7) alone define a $d$-dimensional "Pauli hypercube" $\mathcal{C}^{(d)}=[0,1]^{d}$. Accounting for the ordering and normalization of the natural occupation numbers, $\vec{\lambda}$ is then restricted to the Pauli simplex,

$$
\Sigma^{(N, d)} \equiv\left\{\vec{\lambda} \mid 1 \geqslant \lambda_{1} \geqslant \cdots \geqslant \lambda_{d} \geqslant 0, \sum_{i=1}^{d} \lambda_{i}=N\right\},
$$

which is a $(d-1)$-dimensional subset of the hypercube $\mathcal{C}^{(d)}$. It contains the polytope $\mathcal{P}^{(N, d)}$ as a proper subset, confirming the incompleteness of Pauli's exclusion principle. For an illustration, see Fig. 1. In the following, we will typically skip the indices $N$ and $d$.

\section{THE PINNING PHENOMENON}

The potential influence of the fermionic exchange symmetry beyond Pauli's exclusion principle is strongly linked to the boundary of the polytope corresponding to the saturation of a generalized Pauli constraint. First, it had been argued [11,12] that the ground-state minimization process of the energy expectation value $\langle\Psi|\hat{H}| \Psi\rangle$ for a model Hamiltonian $\hat{H}$ may get stuck on the boundary of the polytope $\mathcal{P}$, since any further minimization would violate some generalized Pauli constraints. This pinning effect by generalized Pauli constraints is physically relevant because it potentially restricts the dynamics of the corresponding system whose natural occupation numbers $\vec{\lambda}$ can never leave the polytope $[13,14]$.
This is in close analogy to the implications of the Pauli constraints which forbid fermions at low temperature to decay to lower-lying occupied energy levels. Second, pinning as an effect in the one-particle picture allows one to reconstruct the structure of the corresponding $N$-fermion quantum state which in addition is significantly simplified.

Let us explain the important latter point. For given $(N, d)$, an arbitrary $N$-fermion state $|\Psi\rangle$ can be expressed as a superposition of $\left(\begin{array}{c}d \\ N\end{array}\right)$ Slater determinants $|\boldsymbol{i}\rangle \equiv a_{i_{1}}^{\dagger} \ldots a_{i_{N}}^{\dagger}|0\rangle$, built from its own natural orbitals $\left|i_{v}\right\rangle$, the eigenstates of the corresponding one-particle reduced density matrix. Now, let us assume that $\vec{\lambda}$ is pinned by a generalized Pauli constraint $D_{j}$, i.e., $D_{j}(\vec{\lambda})=0$. Then, the corresponding superposition is reduced to a subset $\mathcal{I}_{D_{j}}$ containing only those configurations $\boldsymbol{i}$ for which $\hat{D}_{j}|\boldsymbol{i}\rangle=0$ [11]. $\hat{D}_{j}$ follows from $D_{j}$ [Eq. (3)] by replacement of $\lambda_{i}$ by the occupation operator $\hat{n}_{i}$ of the natural orbital $|i\rangle$. Accordingly, the general superposition of $\left({ }_{N}^{d}\right)$ Slater determinants is reduced to (see also right side of Fig. 3)

$$
\left|\Psi^{(\mathrm{pin})}\right\rangle=\sum_{i \in \mathcal{I}_{D_{j}}} c_{i}|\boldsymbol{i}\rangle
$$

This is quite similar to pinning by a Pauli constraint, i.e., $\lambda_{i}=1$ or $\lambda_{i}=0$. In that case, only those Slater determinants $|i\rangle$ contribute to $|\Psi\rangle$ which all do or all do not contain $i$, respectively.

Analytical [14-17] and numerical [18-23] investigations have shown that pinning of the corresponding ground-state natural occupation numbers $\vec{\lambda}$ to the polytope's boundary does not occur, in general. Whereas pinning has been found for some model systems [14,19-21], other systems exhibit quasipinning only $[15,16,18-20]$. In the latter case, the vector of natural occupation numbers $\vec{\lambda}$ is close to, but not exactly on, the polytope's boundary and various implications of pinning hold at least approximately [24].

\section{HIERARCHY OF PINNING}

A crucial point is that the intersection of the polytope's boundary $\partial \mathcal{P}$ and the boundary $\partial \Sigma$ of the Pauli simplex is not empty. In the following, we will show that the set of intersection points exhibits a hierarchical structure which in particular describes the redundancy of the incomplete Pauli constraints given the more restrictive generalized Pauli constraints. As a consequence of the inclusion relation $\mathcal{P} \subset \Sigma$, already observed but not further elaborated in [19], one finds for $\vec{\lambda} \in \mathcal{P}$ that $\vec{\lambda} \in \partial \Sigma \Rightarrow \vec{\lambda} \in \partial \mathcal{P}$. In particular, this applies to the specific part of the boundary of $\Sigma$ corresponding to pinning by some Pauli constraints. However, since $\mathcal{P}$ is not only defined by generalized Pauli constraints but also by ordering constraints $\lambda_{i}-\lambda_{i+1} \geqslant 0, \vec{\lambda} \in \partial \mathcal{P}$ does not always correspond to pinning by generalized Pauli constraints. To explore this in more detail, we express the sets of Pauli constraints in close analogy to Eq. (3) in the compact form

$$
S_{r, s}(\vec{\lambda}) \equiv \sum_{i=1}^{r}\left(1-\lambda_{i}\right)+\sum_{j=d+1-s}^{d} \lambda_{j} \geqslant 0,
$$


with $r \leqslant N, s \leqslant d-N, r+s>0$, and introduce the corresponding simplicial facets of $\Sigma$,

$$
\begin{aligned}
& \Sigma_{r, s} \equiv\left\{\vec{\lambda} \in \Sigma \mid S_{r, s}(\vec{\lambda})=0\right\} \\
& \begin{array}{c}
\left.\Sigma\right|_{\lambda_{1}=\cdots=\lambda_{r}=1} . \\
\lambda_{d+1-s}=\cdots=\lambda_{d}=0
\end{array} .
\end{aligned}
$$

Clearly, there is an inclusion relation for those facets: $\Sigma_{N, d-N}$ is a zero-dimensional simplex, coinciding with the HartreeFock point $\vec{\lambda}_{H F} \equiv(1, \ldots, 1,0, \ldots) . \Sigma_{N, d-N}$ is contained in the next larger facet, $\Sigma_{N-1, d-N-2}$, which is a one-dimensional simplex [25]. This continues up to the largest two facets $\Sigma_{1,0}$ and $\Sigma_{0,1}$, both of dimension $d-2$. In general, we have

$$
\Sigma_{r, s} \subseteq \Sigma_{r^{\prime}, s^{\prime}} \Leftrightarrow r \geqslant r^{\prime} \wedge s \geqslant s^{\prime} .
$$

Similar to $\vec{\lambda} \in F_{j}$, pinning by some Pauli constraints, i.e., $\vec{\lambda} \in \Sigma_{r, s}$, implies strong structural simplifications for the corresponding $N$-fermion state: $r$ fermions are frozen in the first $r$ natural orbitals $|1\rangle, \ldots,|r\rangle$ and the last $s$ natural orbitals $|d-s+1\rangle, \ldots,|d\rangle$ are inactive, i.e.

$$
|\Psi\rangle=a_{1}^{\dagger} \ldots a_{r}^{\dagger}\left|\Psi^{\prime}\right\rangle
$$

The $(N-r)$-fermion state $\left|\Psi^{\prime}\right\rangle$ lives in the corresponding $a c$ tive space, $\left|\Psi^{\prime}\right\rangle \in \wedge^{N-r}\left[\mathcal{H}_{1}^{(d-r-s)}\right]$, where $\mathcal{H}_{1}^{(d-r-s)}$ is spanned by the active orbitals $|r+1\rangle, \ldots,|d-s\rangle$.

From a geometrical viewpoint, for any pair $(r, s)$, pinning by the corresponding Pauli constraints (7) implies pinning to the facet $F_{j}$ of a given generalized Pauli constraint $D_{j}$ if and only if

$$
\mathcal{P} \cap \Sigma_{r, s} \subset F_{j} .
$$

It is worth noticing that $\mathcal{P} \cap \Sigma_{r, s}$ coincides with the polytope $\mathcal{P}^{\prime}$ for the setting $\left(N^{\prime}, d^{\prime}\right) \equiv(N-r, d-r-s)$ (see, e.g., Ref. [15]). In other words, Eq. (11) states that $\forall \vec{\lambda}^{\prime} \in \mathcal{P}^{\prime}$,

$$
D_{j}(\underbrace{1, \ldots, 1}_{r}, \vec{\lambda}^{\prime}, \underbrace{0, \ldots, 0}_{s})=0 .
$$

Based on these observations, natural classes $\mathcal{C}_{r, s}$ of generalized Pauli constraints arise,

$$
\mathcal{C}_{r, s} \equiv\left\{D_{j} \mid \mathcal{P} \cap \Sigma_{r, s} \subset F_{j}\right\} .
$$

The class $\mathcal{C}_{r, s}$ contains exactly those generalized Pauli constraints that are saturated whenever the corresponding Pauli constraints (7) are saturated. Consequently, the classes $\mathcal{C}_{r, s}$ describe the redundancy of the incomplete Pauli constraints given the more restrictive generalized Pauli constraints. Furthermore, the hierarchy (9) implies a hierarchy for these classes,

$$
\mathcal{C}_{r, s} \subseteq \mathcal{C}_{r^{\prime}, s^{\prime}} \Leftrightarrow r \leqslant r^{\prime} \wedge s \leqslant s^{\prime},
$$

and in that sense a partial ordering on $\{(r, s)\}$. By the use of a linear program, we determine all classes and calculate for each generalized Pauli constraint $D_{j}$ all its minimal $(r, s)$ with respect to this partial ordering, i.e., the smallest pairs $(r, s)$ such that $D_{j}$ still belongs to the corresponding class $\mathcal{C}_{r, s}$. The results are listed in the Supplemental Material [26] for the three cases $(N, d)=(3,10),(4,10),(5,10)$.

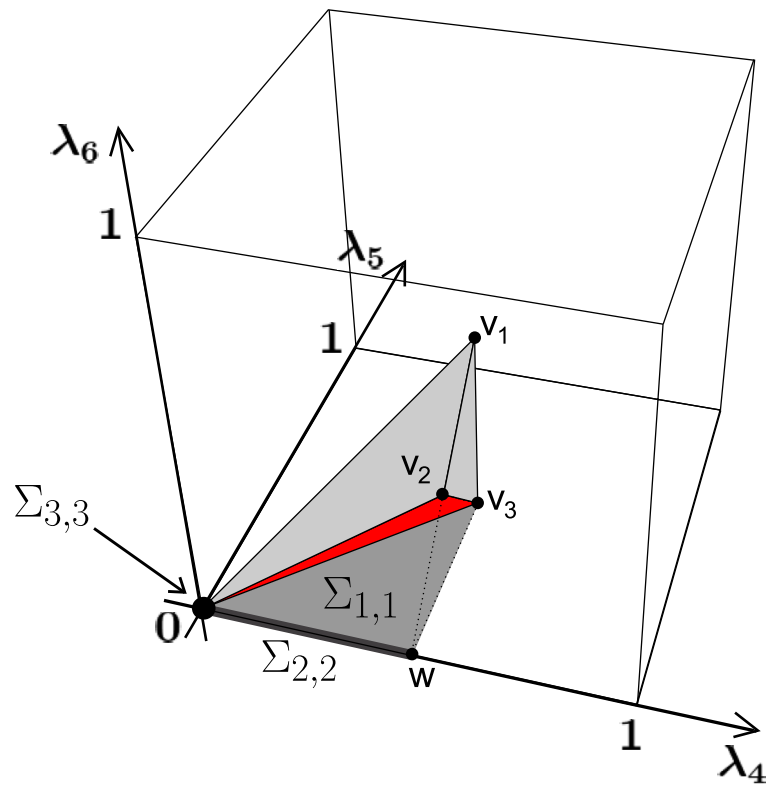

FIG. 2. For the Borland-Dennis setting, $(N, d)=(3,6)$, the reduced polytope of possible spectra $\left(\lambda_{4}, \lambda_{5}, \lambda_{6}\right)$ is shown [the other three natural occupation numbers follow from Eqs. (16)]. It is spanned by the vertices $v_{H F}=(0,0,0), v_{1}=(1 / 2,1 / 2,1 / 2)$, $v_{2}=(1 / 2,1 / 4,1 / 4)$, and $v_{3}=(1 / 2,1 / 2,0)$. Generalized Pauli constraint (17) takes the form $\lambda_{5}+\lambda_{6}-\lambda_{4} \geqslant 0$ and the Pauli simplex $\Sigma$ is spanned by the vertices $v_{H F}, v_{1}, v_{3}$, and $w$.

\section{EXAMPLE FOR THE HIERARCHY OF PINNING}

We illustrate the hierarchy of simplicial facets of the Pauli simplex $\Sigma$ and the induced hierarchy of generalized Pauli constraints for the particular example of the so-called BorlandDennis setting, $(N, d)=(3,6)$. The corresponding polytope is defined by the constraints [7]

$$
\begin{gathered}
\lambda_{1} \geqslant \lambda_{2} \geqslant \lambda_{3} \geqslant \lambda_{4} \geqslant \lambda_{5} \geqslant \lambda_{6} \geqslant 0, \\
\lambda_{1}+\lambda_{6}=\lambda_{2}+\lambda_{5}=\lambda_{3}+\lambda_{4}=1, \\
D(\vec{\lambda}) \equiv 2-\left(\lambda_{1}+\lambda_{2}+\lambda_{4}\right) \geqslant 0 .
\end{gathered}
$$

It is quite special that some generalized Pauli constraints take the form of equalities. Due to Eq. (16), we can choose $\lambda_{4}, \lambda_{5}, \lambda_{6}$ as the independent variables and present the corresponding reduced polytope of possible vectors $\left(\lambda_{4}, \lambda_{5}, \lambda_{6}\right)$ in Fig. 2 . Also various facets of the Pauli simplex are shown: The smallest one is the zero-simplex $\Sigma_{3,3}$ given by the Hartree-Fock point $\left(\lambda_{4}, \lambda_{5}, \lambda_{6}\right)=(0,0,0)$. It is contained in the next larger facet, the one-simplex $\Sigma_{2,2}$ defined by $\lambda_{1}=\lambda_{2}=1, \lambda_{5}=\lambda_{6}=0$. $\Sigma_{2,2}$ is then contained in the largest facet, $\Sigma_{1,1}$, a two-simplex shown in gray and defined by $\lambda_{6}=0$ and $\lambda_{5} \leqslant \lambda_{4} \leqslant \frac{1}{2}$. Notice that it does not make sense to consider the other facets $\Sigma_{r, s}$ with $r \neq s$ since we used the explicit equations (16) to reduce the polytope to three dimensions. The only generalized Pauli constraint which takes the form of a proper inequality is given in this reduced description by $\lambda_{5}+\lambda_{6}-\lambda_{4} \geqslant 0$. The facet $F_{D}$ corresponding to pinning is spanned by the Hartree-Fock point and the vertices $v_{2}, v_{3}$ and is shown in red. We can now determine whether pinning by this generalized Pauli constraint 
is induced by pinning by some Pauli constraints. First, we observe $\Sigma_{3,3} \subset F_{D}$, i.e., pinning by all Pauli constraints implies pinning of the generalized Pauli constraint (17). Next, we consider the smaller pair $(r, s)=(2,2)$. Clearly, whenever $\vec{\lambda} \in \mathcal{P}$ lies in $\Sigma_{2,2}$, it also lies in the red facet (actually $\vec{\lambda}$ has to coincide with the Hartree-Fock point). Thus, constraint (17) belongs also to the smaller class $\mathcal{C}_{2,2}$. Does it also belong to the smallest class $\mathcal{C}_{1,1}$ ? Yes, it belongs also to that class since $\vec{\lambda} \in \mathcal{P} \cap \Sigma_{1,1}$ geometrically implies that $\vec{\lambda}$ lies on the line between the Hartree-Fock point and the polytope vertex $v_{3}$. This line is an edge of the pinning facet $F_{D}$. Hence, the minimal pair $(r, s)$ for generalized Pauli constraint $(17)$ is $(r, s)=(1,1)$.

\section{THE MEASURE}

Since pinning by Pauli constraints implies pinning by specific generalized Pauli constraints according to Eq. (11), the same also holds for quasipinning. Due to the flat geometry of polytope facets, this implication yields linear upper bounds on the $l^{1}$ distance of $\vec{\lambda}$ to the corresponding polytope facets of the form (see also right side of Fig. 1)

$$
\operatorname{dist}_{1}\left(\vec{\lambda}, F_{j}\right) \leqslant \tilde{c}_{j} \operatorname{dist}_{1}\left(\vec{\lambda}, \Sigma_{r, s}\right)
$$

for all $(r, s)$ with $D_{j} \in \mathcal{C}_{r, s}$. Since we are mainly interested in the values $D_{j}(\vec{\lambda})$, we use $\operatorname{dist}_{1}\left(\vec{\lambda}, F_{j}\right) \propto D_{j}(\vec{\lambda})$ (see Appendix A for more details) to obtain bounds of the form

$$
D_{j}(\vec{\lambda}) \leqslant c_{j} \operatorname{dist}_{1}\left(\vec{\lambda}, \Sigma_{r, s}\right) .
$$

Due to the hierarchy (14), we consider (19) only for the minimal pairs $(r, s)$. The optimal (smallest) prefactors $c_{j}$ for various generalized Pauli constraints and all their minimal $(r, s)$ are determined by a linear program and listed in the Supplemental Material [26].

Equipped with bounds (19), we can now decide whether given quasipinning by a generalized Pauli constraint follows from possible approximate saturation of Pauli constraints or whether it goes beyond that. Whenever $D_{j}(\vec{\lambda})$ is significantly smaller than its upper bound (19), the quasipinning is stronger than one could expect from possible quasipinning by Pauli constraints. Such nontriviality of quasipinning by generalized Pauli constraints, which represents the influence of the exchange symmetry beyond the exclusion principle, is quantified by the ratio of both sides in (19). This naturally motivates the following definition for a measure:

$$
Q_{j}(\vec{\lambda}) \equiv-\log _{10}\left[D_{j}(\vec{\lambda}) / c_{j} \operatorname{dist}_{1}\left(\vec{\lambda}, \Sigma_{r, s}\right)\right] .
$$

For generalized Pauli constraints with more than one minimal $(r, s)$, i.e., more than one independent upper bound (19), we divide $D_{j}(\vec{\lambda})$ in Eq. (20) by the maximum of all upper bounds. By construction, $Q_{j}(\vec{\lambda})$ is non-negative. For given $Q_{j}$, the minimal distance of $\vec{\lambda}$ to the polytope boundary is $10^{Q_{j}}$ times smaller than one could expect from an approximate saturation of Pauli constraints. Therefore, for $Q_{j} \lesssim 1$, quasipinning is rather trivial, and for $Q_{j} \gtrsim 2$, quite nontrivial. For practical applications, we define the overall $Q$ parameter,

$$
Q \equiv \max _{j}\left(Q_{j}\right)
$$

A MATHEMATICA package implementing all $Q$ parameters can be obtained from the authors or may be found in the Supplemental Material [26]. The relation between $Q$ parameters of different settings $(N, d)$ is discussed in Appendix B.

\section{OPERATIONAL MEANING OF $Q$}

In addition to its main purpose of measuring the influence of the fermionic exchange symmetry in the one-particle picture beyond Pauli's exclusion principle, the $Q$ parameter also has an operational meaning. To elaborate on this in more detail, we recall the selection rule (6) of Slater determinants in the case of pinning. This remarkable structural simplification of $N$-fermion quantum states also holds (approximately) in the case of quasipinning. To be more precise, one finds [24,27]

$$
\alpha_{-} D(\vec{\lambda}) \leqslant 1-\left\|\hat{P}_{\mathcal{I}_{D}} \Psi\right\|_{L^{2}}^{2} \leqslant \alpha_{+} D(\vec{\lambda}),
$$

for some $\alpha_{-}, \alpha_{+}>0$, where in general $\hat{P}_{\mathcal{I}}$ is defined as the operator projecting on the space spanned by $\{|\boldsymbol{i}\rangle\}_{\boldsymbol{i} \in \mathcal{I}}$. Estimate (22) also holds for Pauli constraint $S_{r, s}(\vec{\lambda}) \geqslant 0$, where the corresponding set $\mathcal{I}_{S_{r, s}}$ is given by the configurations $\boldsymbol{i}=\left(1, \ldots, r, i_{r+1}, \ldots, i_{N}\right), r<i_{v}<d-s+1$, containing all $1, \ldots, r$ but none of $d+1-s, \ldots, d$. Such estimates of the form (22) emphasize the striking relevance that oneparticle information can have for the description of manyfermion quantum systems. Combining condition (22) for Pauli constraint $S_{r, s}$ with (22) for a generalized Pauli constraint $D_{j} \in \mathcal{C}_{r, s}$ yields

$$
\beta_{-} 10^{-Q_{j}(\vec{\lambda})} \leqslant \frac{1-\left\|\hat{P}_{\mathcal{I}_{D_{j}}} \Psi\right\|_{L^{2}}^{2}}{1-\left\|\hat{P}_{\mathcal{I}_{S_{r, s}}} \Psi\right\|_{L^{2}}^{2}} \leqslant \beta_{+} 10^{-Q_{j}(\vec{\lambda})} .
$$

To explain the significance of estimate (23), recall that pinning by generalized Pauli constraints and Pauli constraints is in a one-to-one correspondence to the structures (6) and (10), respectively, with corresponding $\mathcal{I}$. Since pinning by Pauli constraint $S_{r, s}$ implies pinning by generalized Pauli constraint $D \in \mathcal{C}_{r, s}$, we have $\mathcal{I}_{S_{r, s}} \subseteq \mathcal{I}_{D_{j}}$. Therefore, a given $N$-fermion quantum state with natural occupation numbers $\vec{\lambda}$ can be written as

$$
|\Psi\rangle \equiv\left|\Psi_{S}\right\rangle+\left|\Psi_{D \backslash S}\right\rangle+\left|\Psi_{R}\right\rangle,
$$

with $\left|\Psi_{S}\right\rangle \equiv \hat{P}_{\mathcal{I}_{S_{r, s}}}|\Psi\rangle,\left|\Psi_{D \backslash}\right\rangle \equiv \hat{P}_{\mathcal{I}_{D_{j}} \backslash \mathcal{I}_{S_{r, s}}}|\Psi\rangle$, and $\left|\Psi_{R}\right\rangle \equiv$ $\hat{P}_{\mathcal{I}_{D_{j}}^{C}}|\Psi\rangle$, where $\mathcal{I}_{D_{j}}^{C}$ denotes the complement of $\mathcal{I}_{D_{j}}$. Estimate (23) then relates the $L^{2}$ weights of $\left|\Psi_{D \backslash S}\right\rangle$ and $\left|\Psi_{R}\right\rangle$. This is illustrated in Fig. 3. On the left of Fig. 3, only quasipinning by Pauli constraints is considered, which implies that most of the $L^{2}$ weight of $|\Psi\rangle$ is covered by the configurations $i \in \mathcal{I}_{S_{r, s}}$. However, given additional nontrivial quasipinning by a generalized Pauli constraint $D_{j} \in \mathcal{C}_{r, s},|\Psi\rangle$ can be further specified since almost all weight outside $\mathcal{I}_{S_{r, s}}$ needs to lie in $\mathcal{I}_{D_{j}}$ (shown in the middle of Fig. 3). This means that whenever quasipinning by generalized Pauli constraints exceeds quasipinning by Pauli constraints, Eq. (23) allows one to find much more accurate approximations for $|\Psi\rangle$ than by just freezing $r$ electrons in orbitals $|1\rangle, \ldots,|r\rangle$ and omitting $s$ virtual orbitals. This latter case of "large" $Q$ includes the specific case $r=s=0$, i.e., no approximate saturation of any Pauli constraint (illustrated on the right of Fig. 3). Note 

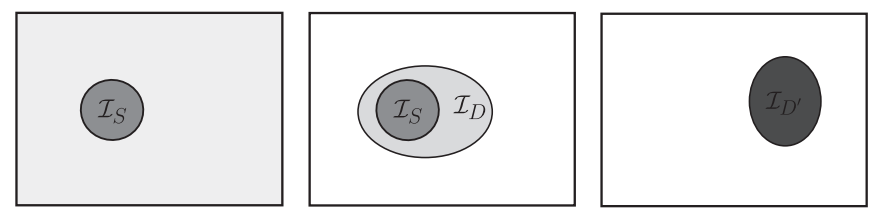

FIG. 3. Schematic illustration of how the $L^{2}$ weight of $|\Psi\rangle$ is distributed over various configurations. Higher weight is indicated by darker colors. Left: We use only the information of quasipinning by Pauli constraints. Middle: Stronger implications follow if additional nontrivial quasipinning by generalized Pauli constraints is taken into account. Right: Even if none of the Pauli constraints is (approximately) saturated, strong quasipinning by generalized Pauli constraints leads to structural simplifications (see text).

also that depending on the corresponding generalized Pauli constraint $D^{\prime}$, the set $\mathcal{I}_{D^{\prime}}$ of possible configurations might be even smaller than the set $\mathcal{I}_{S}$ illustrated on the left and in the middle of Fig. 3.

\section{PROOF OF PRINCIPLE: APPLICATION TO A SIMPLE MODEL}

By applying the $Q$ parameter to a simple model, we provide a proof of principle. This will not only emphasize the significance of our developed concepts but also conclusively confirm that the fermionic exchange symmetry has a relevance in the one-particle picture beyond Pauli's 90-year-old exclusion principle. We consider the same simple model as in Ref. [15],

$H_{N}=\sum_{i=1}^{N}\left(\frac{\vec{p}_{i}^{2}}{2 m}+\frac{m}{2} \vec{x}_{i}^{t} \Omega \vec{x}_{i}\right)+\frac{K}{2} \sum_{1 \leqslant i<j \leqslant N}\left(\vec{x}_{i}-\vec{x}_{j}\right)^{2}$,

where $\vec{x} \in \mathbb{R}^{n}, n \in \mathbb{N}, \Omega \equiv \operatorname{diag}\left(\omega_{1}^{2}, \ldots, \omega_{n}^{2}\right)$, and $\vec{p}_{i} \equiv \frac{\hbar}{i} \vec{\nabla}$.

This analytically solvable $N$-harmonium model has quite a long tradition in physics. Yet, its suggested potential significance for concrete systems as, e.g., for quantum dots [28] is of secondary importance here.

As dimensionless coupling strengths for the different dimensions $i=1, \ldots, n$, we introduce $\kappa_{i}=\frac{N K}{m \omega_{i}^{2}}$. Due to a duality of natural occupation numbers, observed in Refs. [15,29-31] and proven in Ref. [32], we restrict to $\kappa_{i}>0$. We consider the exemplary case of $N=3$ fermions in $n=2$ spatial dimensions. The corresponding coupling regime can be parameterized by $\kappa \equiv \kappa_{1}$ and $\omega_{2} / \omega_{1}$ and the results for the ground state are shown in Fig. 4. There, the solid black line denotes the crossing of the ground state and the first-excited state and dashed lines indicate crossing of natural occupation numbers, which can change the quasipinning behavior as well [14]. From the left of Fig. 4, we learn that quasipinning by generalized Pauli constraints becomes stronger whenever the coupling $\kappa$ decreases and the trap becomes more anisotropic. However, the $Q$ parameter presented on the right of Fig. 4 shows that the phenomenon of quasipinning is much more involved than previously appreciated. For instance, the strong quasipinning in the regime $\kappa \omega_{2} / \omega_{1} \approx 10$ is completely trivial, i.e., it is an immediate consequence of quasipinning by Pauli constraints. It is surrounded from both sides by regimes of highly nontrivial quasipinning by generalized Pauli constraints. Similar results
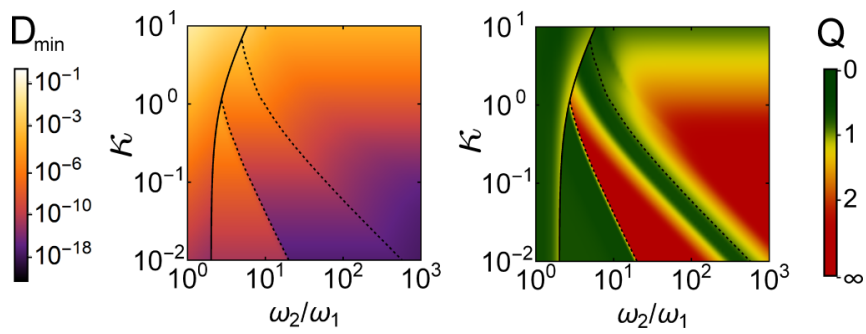

FIG. 4. Quasipinning results for a three-harmonium ground state in two dimensions with external trap frequencies $\omega_{1}, \omega_{2}$ and coupling strength $\kappa$. Minimal distance $D_{\min }$ of $\vec{\lambda}$ to the polytope boundary is shown on the left and its nontriviality is quantified on the right (see text for details).

are found also for larger $N$ and larger dimensions [16,17]. This conclusively confirms the physical relevance of the generalized Pauli constraints (recall also Sec. III) and shows that the fermionic exchange symmetry can have an influence on the one-particle picture beyond Pauli's exclusion principle for concrete systems.

\section{SUMMARY AND CONCLUSION}

We have explored whether the fermionic exchange symmetry - a property on the $N$-particle level — can have an influence on the one-particle picture beyond Pauli's exclusion principle. By exploiting a geometric picture, we first explored and described in the form of a pinning hierarchy the redundancy of Pauli's incomplete exclusion principle given the more restrictive generalized Pauli constraints. Based on this hierarchy, we succeeded in constructing a measure (" $Q$ parameter") which allows one to quantify for concrete systems the influence of the generalized Pauli constraints beyond the Pauli exclusion principle constraints.

From an operational viewpoint, the $Q$ parameter has an even more important additional significance, since it quantifies possible structural simplifications for $N$-fermion quantum states beyond the active space size. In particular, this provides a perspective on fermionic multipartite correlation since it allows the distinction between static and dynamic correlation. To explain this, we first need to discuss the meaning of the Pauli constraints $S_{r, s}(\vec{\lambda}) \geqslant 0$ [Eq. (7)]. The value $S_{r, s}(\vec{\lambda})$ represents the weight of the corresponding $N$ fermion quantum state $|\Psi\rangle$ outside the active space obtained by freezing $r$ electrons (in the first $r$ natural orbitals) and skipping $s$ virtual states (the last $s$ natural orbitals). As a consequence, $S_{r, s}(\vec{\lambda})$ quantifies [27] the numerical accuracy of the "complete active space self-consistent field" method (see, e.g., Ref. [33]) using the corresponding active space. Since so-called dynamical correlations (see, e.g., Ref. [34]) are attributed to a large number of Slater determinants contributing with small amplitudes to $|\Psi\rangle$, the values $\left\{S_{r, s}(\vec{\lambda})\right\}$ measure dynamical correlations. Indeed, if $S_{r, s}(\vec{\lambda}) \approx 0$ for some $r, s$ sufficiently close to $N$ and $d-N$, respectively, almost all weight of $|\Psi\rangle$ has to lie in a small active space. In contrast to the values $\left\{S_{r, s}(\vec{\lambda})\right\}$, the parameters $Q_{j}$ describe how the weight of the $N$-fermion quantum state distributes over configurations within the different active spaces. This is illustrated in Fig. 3. On the left side (corresponding to a small 
$Q_{j}$ ), all configurations may in principle contribute to $|\Psi\rangle$, but with small amplitudes (dynamic correlations). This is quite different to the middle of Fig. 3, where "large" $Q_{j}$ implies that the weight shrinks to only a few configurations, reflecting the presence of "static correlations" [34]. Since the type of correlations present in a given system determines whether a multireference or single-reference approach is required, the $Q_{j}$ parameters may prove useful as an additional tool for this important decision.

By applying the $Q$ parameter to a simple model, we provided a proof of principle for the relevance of our concepts. Our results conclusively confirm the physical relevance of the generalized Pauli constraints (recall also Sec. III) and show that the fermionic exchange symmetry can have an influence on the one-particle picture beyond Pauli's exclusion principle for concrete systems. Our findings also suggest that the phenomenon of quasipinning by generalized Pauli constraints is much more involved than previously appreciated. Equipped with our measure $Q$, the ongoing debate on the relevance of generalized Pauli constraints may change significantly after reconsidering and reanalyzing [11,14,15,18-22,24,35-38]. Indeed, most of those papers have not contrasted their respective findings with the less restrictive Pauli exclusion principle constraints and have therefore confirmed the physical relevance of the generalized Pauli constraints for the wrong reasons.

Last but not least, our work can be regarded as the starting point of a research avenue based on Klyachko's breakthrough result solving the one-body pure $N$-representability problem: the systematic analysis and quantification of the influence of the fermionic exchange symmetry beyond the well-established influence of Pauli's exclusion principle, possibly also in the more-particle picture.

\section{ACKNOWLEDGMENTS}

We thank C.Benavides-Riveros, M.Christandl, D.Ebler, D.Gross, D.Jaksch, and B.Yadin for helpful discussions. We gratefully acknowledge financial support from the FriedrichNaumann-Stiftung and Christ Church Oxford (F.T.), the Oxford Martin School, the NRF (Singapore), the MoE (Singapore), the EU Collaborative Project TherMiQ (Grant Agreement No. 618074) (V.V.), the Swiss National Science Foundation (Grant No. P2EZP2 152190), the Oxford Martin Programme on Bio-Inspired Quantum Technologies, and the UK Engineering and Physical Sciences Research Council (Grant No. EP/P007155/1) (C.S.).

\section{APPENDIX A: DISTANCE OF $\vec{\lambda}$ TO POLYTOPE FACETS}

Let us first consider the restriction of the Euclidean space $\mathbb{R}^{d}$ to the hyperplane $V$,

$$
V \equiv\left\{\vec{x} \in \mathbb{R}^{d} \mid x_{1}+\cdots+x_{d}=N\right\},
$$

where $N$ is the particle number of the setting of interest. Below, we will restrict these considerations to proper spectra $\vec{x}=$ $\left(x_{i}\right)_{i=1}$, i.e., to non-negative and decreasingly ordered entries $x_{i}$. Consider now a hyperplane in $V$ defined by

$$
E_{D} \equiv\left\{\vec{x} \in V \mid D(\vec{x}) \equiv \kappa^{(0)}+\kappa^{(1)} x_{1}+\cdots+\kappa^{(d)} x_{d}=0\right\} .
$$

We are interested in deriving an explicit expression for the $l^{1}$ distance of $\vec{y} \in V$ to the hyperplane $E_{D} \subset V$. For this, we express the distance of $\vec{y}$ to a set $A \subset V$ as

$$
\operatorname{dist}_{1}(\vec{y}, A)=\sup \left(\left\{r \mid \mathcal{B}_{1}^{(r)}(\vec{y}) \cap A=\emptyset\right\}\right),
$$

where

$$
\mathcal{B}_{1}^{(r)}(\vec{y}) \equiv\left\{\vec{x} \in V \mid\|\vec{x}-\vec{y}\|_{1} \leqslant r\right\}
$$

is the $l^{1}$ Ball with radius $r>0$ around $\vec{y}$ and $\|\vec{x}\|_{1} \equiv \sum_{i=1}^{d}\left|x_{1}\right|$ denotes the $l^{1}$ norm. Obviously, $\mathcal{B}_{1}^{(r)}(\vec{y})$ has the shape of a cube intersected with the hyperplane $x_{1}+\cdots+x_{d}=N$, i.e., it is in particular a polytope and has therefore finitely many extremal points, called vertices.

For $\vec{y} \notin A$, it is instructive to think of (A3) as the radius $r_{0}$ that one obtains by further increasing the originally very small radius of $\mathcal{B}_{1}^{(r)}(\vec{y})$ until one of its points reaches $A$. For $A=E_{D}$, we observe that in such a process, $E_{D}$ is first reached by a vertex of $\mathcal{B}_{1}^{(r)}(\vec{y})$. This follows from the fact that $E_{D}$ is flat. As an elementary exercise, one can determine all the vertices of $\mathcal{B}_{1}^{(r)}(\vec{y})$, following as

$$
\vec{v}_{i, j, \pm}^{(r)}(\vec{y})=\vec{y} \pm \frac{r}{2}\left(\vec{e}_{i}-\vec{e}_{j}\right),
$$

where $i, j=1,2, \ldots, d$ with $i<j$, and $\vec{e}_{j}$ is a unit vector in the $j$ direction. Hence, to determine $r_{0} \equiv \operatorname{dist}_{1}\left(\vec{y}, E_{D}\right)$, we need to find roots $r \geqslant 0$ of

$$
D\left(\vec{v}_{i, j, \sigma}^{(r)}(\vec{y})\right)=D(\vec{y})+\sigma \frac{r}{2}\left(\kappa^{(i)}-\kappa^{(j)}\right)=0
$$

for various $i, j=1,2, \ldots, d$ with $i<j$ and $\sigma= \pm$. $r_{0}$ will then be given by the minimum of all those solutions. For a consistency check, notice that $r=0$ solves (A6) for $\vec{y} \in E_{D}$ independent of $i, j, \sigma$, and thus $\operatorname{dist}_{1}\left(\vec{y}, E_{D}\right)=0$. If $\vec{y} \notin E_{D}$, we have $D(\vec{y}) \neq 0$. For $i, j$ with $\kappa^{(i)}=\kappa^{(j)}$, there is no solution of (A6). For $i, j$ with $\kappa^{(i)} \neq \kappa^{(j)}$, we find a solution $r>0$ whenever $\sigma$ has the correct sign:

$$
r=\left|\frac{2 D(\vec{y})}{\kappa^{(i)}-\kappa^{(j)}}\right| .
$$

Finally, this leads to

$$
\operatorname{dist}_{1}\left(\vec{y}, E_{D}\right) \equiv r_{0}=\frac{2 D(\vec{y})}{\Delta \kappa_{\text {max }}}, \quad \forall \vec{y} \in V,
$$

where

$$
\Delta \kappa_{\max }=\max _{i, j}\left\{\left|\kappa_{i}-\kappa_{j}\right|\right\} .
$$

In particular, the result (A8) holds for $D$ a generalized Pauli constraint and for all $\vec{y} \in V$, and therefore in particular also for all $\vec{y} \in \mathcal{P} \subset V$. By restricting the hyperplane $E_{D}$ to the polytope $\mathcal{P}$ of possible spectra $\vec{\lambda} \equiv\left(\lambda_{i}\right)_{i=1}^{d}$ (i.e., in particular to decreasingly ordered and non-negative $\lambda_{i}$ ), we obtain a facet $F_{D}$ of that polytope. The $l^{1}$ distance of $\vec{\lambda} \in \mathcal{P}$ to $F_{D}$ is identical to the expression (A8) only for those $\vec{\lambda}$ whose minimal distance to $E_{D}$ is attained in the subset $F_{D} \subset E_{D}$. However, this is always the case for $\vec{\lambda} \in \mathcal{P}$ sufficiently close to $F_{D}$ and $E_{D}$, respectively. To summarize, whenever $\vec{\lambda} \in \mathcal{P}$ is sufficiently strongly quasipinned to a facet $F_{D}$, its $l^{1}$ distance 
is related to $D(\vec{\lambda})$ by

$$
\operatorname{dist}_{1}\left(\vec{\lambda}, F_{D}\right)=\frac{2 D(\vec{\lambda})}{\Delta \kappa_{\max }} .
$$

For the case that this distance is not small enough, i.e., in particular larger than the distance of $\vec{\lambda}$ to another facet $F^{\prime}$ of $\mathcal{P}$ adjacent to $F_{D}$, the right-hand side of (A10) needs to be modified by an additional prefactor larger than one. However, it is important to keep in mind that the value $D(\vec{\lambda})$ is the relevant quantity. Still, for didactic reasons, it is instructive and convenient to talk about the polytope $\mathcal{P}$ formed by all possible spectra $\vec{\lambda}$ and relate $D(\vec{\lambda})$ to the distance of $\vec{\lambda}$ to the corresponding polytope facet $F_{D}$ rather than to $E_{D}$.

\section{APPENDIX B: RELATION BETWEEN $Q$ PARAMETERS OF DIFFERENT SETTINGS}

In this section, we briefly recall the required concept of truncation and comment on the relation between $Q$ parameters of different settings. We consider two different settings, $(N, d)$ and $\left(N^{\prime}, d^{\prime}\right) \equiv(N-\Delta N, d-\Delta d)$. Their polytopes $\mathcal{P}$ and $\mathcal{P}^{\prime}$ are strongly related according to Ref. [35],

$$
\mathcal{P}^{\prime}=\mathcal{P} \cap \Sigma_{\Delta N, \Delta d-\Delta N} .
$$

This means that intersecting the polytope $\mathcal{P}$ of the larger setting with the hyperplane defined by

$\lambda_{1}=\cdots=\lambda_{\Delta N}=1 \wedge \lambda_{d+1-\Delta d+\Delta N}=\cdots=\lambda_{d}=0$

yields the polytope $\mathcal{P}^{\prime}$ (which is, of course, still embedded in $\mathbb{R}^{d}$ ). Relation (B1) means that for every generalized Pauli constraint $D^{\prime}$ of the smaller setting, there exists at least one corresponding generalized Pauli constraint $D$ of the larger setting, an extension of $D^{\prime}$, i.e.,

$$
\begin{aligned}
D(\vec{\lambda})= & \sum_{i=1}^{\Delta N} \tilde{\kappa}_{i}\left(1-\lambda_{i}\right)+D^{\prime}\left(\lambda_{\Delta N+1}, \ldots, \lambda_{d-\Delta d+\Delta N}\right) \\
& +\sum_{j=d+1-\Delta d+\Delta N}^{d} \kappa_{j} \lambda_{j} .
\end{aligned}
$$

Equation (B3) means simply

$$
D^{\prime}\left(\vec{\lambda}^{\prime}\right)=D(\underbrace{1, \ldots, 1}_{\Delta N}, \vec{\lambda}^{\prime}, \underbrace{0, \ldots, 0}_{d-\Delta d+\Delta N}) .
$$

For all remaining generalized Pauli constraints $D$ of $(N, d)$ without a partner generalized Pauli constraint in $\left(N^{\prime}, d^{\prime}\right)$, their restriction to the hyperplane (B2) leads to an inequality in the remaining natural occupation numbers $\vec{\lambda}^{\prime}$ which linearly depends on the generalized Pauli constraints of $\left(N^{\prime}, d^{\prime}\right)$. As a consequence, those generalized Pauli constraints $D$ can be neglected whenever the first $\Delta N$ natural occupation numbers are sufficiently close to 1 and the last $(\Delta d-\Delta N)$ natural occupation numbers are sufficiently close to 0 .

The concept of a truncated quasipinning analysis follows from those observations: The analysis of possible quasipinning of given $\vec{\lambda} \in \mathcal{P}$ can be simplified by omitting various natural occupation numbers very close to 1 and 0 and exploring quasipinning for the remaining natural occupation numbers $\vec{\lambda}^{\prime}$ in the truncated polytope $\mathcal{P}^{\prime}$. The minimal distance of $\vec{\lambda}$ to a generalized Pauli constraint facet of $\mathcal{P}$ follows then as the minimal distance of $\vec{\lambda}^{\prime}$ to a generalized Pauli constraint facet of $\mathcal{P}^{\prime}$ up to a small error. This error is given by a linear form in the neglected natural occupation numbers, $1-\lambda_{1}, \ldots, 1-\lambda_{\Delta N}, \lambda_{d+1-\Delta d+\Delta N}, \ldots, \lambda_{d}$. Notice that the concept of truncation allows one to perform a quasipinning analysis even if the polytope $\mathcal{P}$ is not known yet, provided that sufficiently many natural occupation numbers are very close to 1 and 0 , respectively. This then leads to a truncated setting $\left(N^{\prime}, d^{\prime}\right)$, whose polytope might already be known.

The concept of a truncated quasipinning analysis was successfully applied to various states of the $N$-harmonium (25) studied in this work. There, the one-particle Hilbert space is even infinite dimensional. However, since almost all natural occupation numbers are sufficiently close to 0 , we could truncate the analysis to the settings $(3,10)$, whose generalized Pauli constraints are already known. The corresponding truncation error was checked to be always smaller than various distances of the truncated $\vec{\lambda}^{\prime}$ to the generalized Pauli constraint facets of $\mathcal{P}^{\prime}$.

The concept of truncation also suggests that the $Q$ parameters of different settings are related. One may expect the relation

$$
Q(\underbrace{1^{-}, \ldots, 1^{-}}_{\Delta N}, \vec{\lambda}^{\prime}, \underbrace{0^{+}, \ldots, 0^{+}}_{\Delta d-\Delta N})=Q^{\prime}\left(\vec{\lambda}^{\prime}\right), \forall \vec{\lambda}^{\prime} \in \mathcal{P}^{\prime},
$$

where $1^{-}, 0^{+}$means to perform a corresponding limit (avoiding possible expressions of the form $0 / 0$ ). However, since some of the individual prefactors in (19) may vary slightly for larger settings, (B5) may hold in those cases only approximately. Yet, this would not have any qualitative influence on the conclusion of trivial or nontrivial quasipinning. To elaborate on this, we consider two generalized Pauli constraints $D^{\prime}$ in $\left(N^{\prime}, d^{\prime}\right)$ and $D$ in $(N, d)$ which are related according to Eq. (B3). Relation (B3) then implies

$$
\forall r, s: \quad D^{\prime} \in \mathcal{C}_{r, s}^{\prime} \Leftrightarrow D \in \mathcal{C}_{r+\Delta N, s+\Delta d-\Delta N},
$$

where $\mathcal{C}_{r, s}^{\prime}$ are the classes (13) for the setting $\left(N^{\prime}, d^{\prime}\right)$ and $\mathcal{C}_{r, s}$ those for $(N, d)$. From Eq. (B6), we conclude that a pair $\left(r^{\prime}, s^{\prime}\right)$ minimal (given $D^{\prime}$ still in $\mathcal{C}_{r^{\prime}, s^{\prime}}^{\prime}$ ) is equivalent to $(r, s) \equiv$ $\left(r^{\prime}+\Delta N, s^{\prime}+\Delta d-\Delta N\right)$ minimal (given $D$ still in $\left.\mathcal{C}_{r, s}\right)$. This yields related optimal bounds [recall (18) and (19)],

$$
\begin{gathered}
D^{\prime}\left(\vec{\lambda}^{\prime}\right) \leqslant c^{\prime} \operatorname{dist}_{1}\left(\vec{\lambda}^{\prime}, \Sigma_{r, s}^{\prime}\right), \\
D(\vec{\lambda}) \leqslant c \operatorname{dist}_{1}\left(\vec{\lambda}, \Sigma_{r+\Delta N, s+\Delta d-\Delta N}\right) .
\end{gathered}
$$

Restricting the bound for $D(\vec{\lambda})$ in Eq. (B8) to the hyperplane (B2) yields bound (B7) up to a potentially different prefactor. Indeed, we observe

$$
\begin{aligned}
c & \equiv \min _{\vec{\lambda} \in \mathcal{P}}\left[\frac{D(\vec{\lambda})}{\operatorname{dist}_{1}\left(\vec{\lambda}, \Sigma_{r+\Delta N, s+\Delta d-\Delta N}\right)}\right] \\
& \leqslant \min _{\vec{\lambda} \in \mathcal{P} \cap \Sigma_{\Delta N, \Delta d-\Delta N}}\left[\frac{D(\vec{\lambda})}{\operatorname{dist}_{1}\left(\vec{\lambda}, \Sigma_{r+\Delta N, s+\Delta d-\Delta N}\right)}\right] \\
& =\min _{\vec{\lambda}^{\prime} \in \mathcal{P}^{\prime}}\left[\frac{D^{\prime}\left(\vec{\lambda}^{\prime}\right)}{\operatorname{dist}_{1}\left(\vec{\lambda}^{\prime}, \Sigma_{r, s}^{\prime}\right)}\right] \\
& \equiv c^{\prime},
\end{aligned}
$$


where we used Eqs. (B1), (B3), and (B4) in the second to last line.

Whenever the prefactors $c$ and $c^{\prime}$ are different, the corresponding $Q$ parameters (21), i.e., $Q$ for $D$ and $Q^{\prime}$ for $D^{\prime}$, do not obey Eq. (B5). As a consequence, the overall $Q$ parameters (21) may also not fulfill relation (B5). Yet this would not have any qualitative influence on the conclusion of trivial or nontrivial quasipinning. This is based on the fact that $c$ and $c^{\prime}$ differ only for a few related generalized Pauli constraints $D^{\prime}$ and $D$, and then only by a few percent.

\section{APPENDIX C: EXPLICIT FORM OF THE $Q$ PARAMETER}

The generalized Pauli constraints are known so far only for settings $(N, d)$ with $d \leqslant 10$. We present for the three largest ones, $(N, d)=(3,10),(4,10),(5,10)$, the details of the $Q$ parameter and list them in the Supplemental Material [26]. We expressed the generalized Pauli constraints in the canonical form,

$$
D_{j}(\vec{\lambda}) \equiv \kappa_{j}^{(0)}+\sum_{i=1}^{d} \kappa_{j}^{(i)} \lambda_{i} \geqslant 0,
$$

where $j=1,2, \ldots, v_{N, d}<\infty$. For each generalized Pauli constraint $D_{j}$, we determine by resorting to a linear program their extremal classes $\mathcal{C}_{r, s}(10)$, i.e., the minimal pairs $(r, s)$ provided $D_{j} \in \mathcal{C}_{r, s}$. These pairs $(r, s)$ are presented in the Supplemental Material [26], in the second to last column of the respective tables. There, we decode these pairs in the form $\{r, d+1-s\}$ and also skip possible entries $r=0$ and $d+1-s=d+1$. Notice that a single entry $\{t\}$ then stands for $\{0, t\}$ if $t>N$ and for $\{t, d+1\}$ if $t \leqslant N$. Some generalized Pauli constraints do not belong to any class $\mathcal{C}_{r, s}$, i.e., the corresponding polytope facet does not contain the HartreeFock point. This is indicated by leaving the corresponding entry in the second to last column empty. For some generalized Pauli constraints, there is more than one minimal "pair" (but never more than two). Each such minimal pair gives rise to an upper bound of the form

$$
D_{j}(\vec{\lambda}) \leqslant c_{j} \operatorname{dist}_{1}\left(\vec{\lambda}, \Sigma_{r, s}\right) \quad \forall \vec{\lambda} \in \mathcal{P},
$$

where $\Sigma_{r, s}$ is defined in Eq. (8). By using similar techniques as for the derivation of the $l^{1}$ distance of $\vec{\lambda}$ to the polytope facet $F_{D}$ (cf. Appendix A), one shows

$$
\operatorname{dist}_{1}\left(\vec{\lambda}, \Sigma_{r, s}\right)=2 \max \left[\sum_{i=1}^{r}\left(1-\lambda_{i}\right), \sum_{j=d+1-s}^{d} \lambda_{j}\right] .
$$

The minimal coefficients $c_{j}$ in Eq. (C2) are determined by a linear program and are listed in the last columns of the three tables in the Supplemental Material [26]. For any generalized Pauli constraint $D_{i}$ which does not belong to any class $\mathcal{C}_{r, s}$, we establish an elementary upper bound of the form

$$
D_{i}(\vec{\lambda}) \leqslant c_{i} 1
$$

and the corresponding minimal $c_{i}$ are listed in the tables as well.

Let us consider an example: The seventh generalized Pauli constraint of the setting $(3,10)$ has two independent upper bounds, described by the two minimal pairs $\{r, d+1-s\}=$ $\{7\},\{1,8\}$. The corresponding prefactors follow as 1 and $3 / 4$. This means that the optimal bounds on $D_{7}$ are given by

$$
\begin{aligned}
& D_{7}(\vec{\lambda}) \leqslant 1 \operatorname{dist}_{1}\left(\vec{\lambda}, \Sigma_{0,4}\right) \quad \text { and } \\
& D_{7}(\vec{\lambda}) \leqslant \frac{3}{4} \operatorname{dist}_{1}\left(\vec{\lambda}, \Sigma_{1,3}\right) .
\end{aligned}
$$

According to the definition (14), the corresponding $Q_{7}$ parameter follows as

$$
Q_{7}(\vec{\lambda})=-\log _{10}\left\{\frac{D_{7}(\vec{\lambda})}{\max \left[\operatorname{dist}_{1}\left(\vec{\lambda}, \Sigma_{0,4}\right), \frac{3}{4} \operatorname{dist}_{1}\left(\vec{\lambda}, \Sigma_{1,3}\right)\right]}\right\} .
$$

In the same way, we find, for the third generalized Pauli constraint of the same setting,

$$
Q_{3}(\vec{\lambda})=-\log _{10}\left[\frac{14}{9} D_{3}(\vec{\lambda})\right]
$$

[1] W. Pauli, Über den zusammenhang des abschlusses der elektronengruppen im atom mit der komplexstruktur der spektren, Z. Phys. 31, 765 (1925).

[2] F. J. Dyson, Groundstate energy of a finite system of charged particles, J. Math. Phys. 8, 1538 (1967).

[3] E. H. Lieb, The stability of matter, Rev. Mod. Phys. 48, 553 (1976).

[4] P. A. M. Dirac, On the theory of quantum mechanics, Proc. R. Soc. London A 112, 661 (1926).

[5] W. Heisenberg, Mehrkörperproblem und resonanz in der quantenmechanik, Z. Phys. 38, 411 (1926).

[6] R. B. Laughlin, Anomalous quantum Hall effect: An Incompressible Quantum Fluid with Fractionally Charged Excitations, Phys. Rev. Lett. 50, 1395 (1983).

[7] R. E. Borland and K. Dennis, The conditions on the one-matrix for three-body fermion wave functions with one-rank equal to six, J. Phys. B 5, 7 (1972).
[8] M. Altunbulak and A. Klyachko, The Pauli principle revisited, Commun. Math. Phys. 282, 287 (2008).

[9] A. A. Klyachko, Quantum marginal problem and Nrepresentability, J. Phys.: Conf. Ser. 36, 72 (2006).

[10] M. Altunbulak, The Pauli principle, representation theory, and geometry of flag varieties, Ph.D. thesis, Bilkent University, 2008.

[11] A. A. Klyachko, The Pauli exclusion principle and beyond, arXiv:0904.2009.

[12] A. A. Klyachko, The Pauli principle and magnetism, arXiv:1311.5999.

[13] C. Schilling, The quantum marginal problem, in Mathematical Results in Quantum Mechanics (World Scientific, Singapore, 2015), Chap. 1, pp. 165-176.

[14] C. Schilling, Hubbard model: Pinning of occupation numbers and role of symmetries, Phys. Rev. B 92, 155149 (2015). 
[15] C. Schilling, D. Gross, and M. Christandl, Pinning of Fermionic Occupation Numbers, Phys. Rev. Lett. 110, 040404 (2013).

[16] F. Tennie, D. Ebler, V. Vedral, and C. Schilling, Pinning of fermionic occupation numbers: General concepts and one spatial dimension, Phys. Rev. A 93, 042126 (2016).

[17] F. Tennie, V. Vedral, and C. Schilling, Pinning of fermionic occupation numbers: Higher spatial dimensions and spin, Phys. Rev. A 94, 012120 (2016).

[18] C. L. Benavides-Riveros, J. M. Gracia-Bondia, and M. Springborg, Quasipinning and entanglement in the lithium isoelectronic series, Phys. Rev. A 88, 022508 (2013).

[19] R. Chakraborty and D. A. Mazziotti, Generalized Pauli conditions on the spectra of one-electron reduced density matrices of atoms and molecules, Phys. Rev. A 89, 042505 (2014).

[20] I. Theophilou, N. N. Lathiotakis, M. Marques, and N. Helbig, Generalized Pauli constraints in reduced density matrix functional theory, J. Chem. Phys. 142154108 (2015).

[21] C. L. Benavides-Riveros and M. Springborg, Quasipinning and selection rules for excitations in atoms and molecules, Phys. Rev. A 92, 012512 (2015).

[22] R. Chakraborty and D. A. Mazziotti, Role of the generalized Pauli constraints in the quantum chemistry of excited states, Int. J. Quantum Chem. 116, 784 (2016).

[23] D. A. Mazziotti, Pure- $n$-representability conditions of twofermion reduced density matrices, Phys. Rev. A 94, 032516 (2016).

[24] C. Schilling, Quasipinning and its relevance for $n$-fermion quantum states, Phys. Rev. A 91, 022105 (2015).

[25] Due to the normalization $\lambda_{1}+\cdots+\lambda_{d}=N$, the simplices $\Sigma_{N-1, d-N}$ and $\Sigma_{N, d-N-1}$ coincide with $\Sigma_{N, d-N}$ and we omit them.

[26] See Supplemental Material at http://link.aps.org/supplemental/ 10.1103/PhysRevA.95.022336 for the list of all $Q$-parameters for the settings $(N, d)=(3,10),(4,10),(5,10)$.
[27] C. Benavides-Riveros, C. Schilling, and P. Vrana (unpublished).

[28] N. F. Johnson and M. C. Payne, Exactly Solvable Model of Interacting Particles in a Quantum Dot, Phys. Rev. Lett. 67, 1157 (1991).

[29] J. Pipek and I. Nagy, Measures of spatial entanglement in a two-electron model atom, Phys. Rev. A 79, 052501 (2009).

[30] C. Schilling, Natural orbitals and occupation numbers for harmonium: Fermions versus bosons, Phys. Rev. A 88, 042105 (2013).

[31] M. L. Glasser and I. Nagy, Exact evaluation of entropic quantities in a solvable two-particle model, Phys. Lett. A 377, 2317 (2013).

[32] C. Schilling and R. Schilling, Duality of reduced density matrices and their eigenvalues, J. Phys. A 47, 415305 (2014).

[33] F. Jensen, Introduction to Computational Chemistry (Wiley, New York, 2006)

[34] K. Boguslawski, P. Tecmer, Ö. Legeza, and M. Reiher, Entanglement measures for single- and multireference correlation effects, J. Phys. Chem. Lett. 3, 3129 (2012).

[35] C. Schilling, Quantum marginal problem and its physical relevance, Ph.D. thesis, ETH-Zürich, 2014.

[36] R. Chakraborty and D. A. Mazziotti, Sufficient condition for the openness of a many-electron quantum system from the violation of a generalized Pauli exclusion principle, Phys. Rev. A 91, 010101 (2015).

[37] A. Lopes, Pure univariate quantum marginals and electronic transport properties of geometrically frustrated systems, Ph.D. thesis, University of Freiburg, 2015.

[38] J. Wang and P. J. Knowles, Nonuniqueness of algebraic firstorder density-matrix functionals, Phys. Rev. A 92, 012520 (2015). 